\title{
Health Monitoring System using Raspberry Pi and IOT
}

\author{
E. N. GANESH
}

VISTAS, Chennai, India.

\begin{abstract}
Abstrac
Health Monitoring system using IOT describes the collection and interoperation of Patient data collected from the sensors from the hospitals through IOT Technology. The collected sensor data will support the doctor in the emergency situation for the betterment and improvement of Patient health. The hardware platform to implement the project consists of a sensor and Raspberry Pi 3 Model B equipped in a way to communicate with doctor through Internet and Smart Phone. This proposed idea will help doctors to know about the state of patient health and monitor anywhere in the world. In this proposed idea the sensors gather the medical information of the patient that include patient's heart rate, blood pressure, and pulse rate Then using camera the patient is lively monitored through the Raspberry kit and this information is sent to the Internet and stored in a medical server. The doctor and patient can monitor the patient data from any place of the world through the provided IP server address anytime. The emergency alert is sent to the patient if the sensor value is exceeded the threshold data. Thus the patients health parameters are watched lively and regular monitoring through the medical server to a doctor will help to make effective diagnosis and almost accurate care can be given. The data collection through the IOT will help the patient to recover easily and also enhanced medical care can be given to the patients with low cost.
\end{abstract}

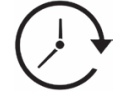

Article History

Received: 30 November 2019 Accepted: 25 february 2019
\end{abstract}

\section{Keywords}

Healthcare;

Health Monitoring;

IoT;

Medical Services.

\section{Introduction}

The Internet of Things is a rising topic of social, economic and technical significance. Internet of Thing using sensors, Processors and Microcontrollers with accessories used for communication through the internet and becoming the constitutive part of the Internet, it is built with a suitable protocol which helps the interacting and communicating with each other and with the users respectively. This communication through internet helps to find many

CONTACT E. N. Ganesh Enganesh50@gmail.com 9 vISTAS, Chennai, India.

\section{(c) (i)}

(C) 2018 The Author(s). Published by Oriental Scientific Publishing Company

This is an Open Access article licensed under a Creative Commons license: Attribution 4.0 International (CC-BY).

Doi: http://dx.doi.org/10.13005/ojcst12.01.03 
applications which are developed based on IOT technology in which every physical object like sensor devices are connected to the internet. ${ }^{1}$ Healthcare plays a major role in the Internet of things which reduces the difficulty faced patients and doctors. The homecare is provided instead of the expensive clinical care and prevention is provided by the efficient healthcare service. This service will help every individual by following the basic healthcare, which leads to more advantageous results. ${ }^{2}$ IOT technology is increasing to support the cost and quality of patient life and also ensures life span of patients with proper medication. In conventional health care undetected health problems can be solved through this loT Technology thereby ensures healthcare services by maintaining digital identity for each patient complication can be greatly reduced. The communication between the health sensors device with the computer or smartphone which has the default ability to communicate with the server which makes the whole system cost reduce and the complexity of the system is also reduced. Hence the system can also be made loT enabled and Machine TO Machine compatible. ${ }^{3}$ Here the proposed paper show a reliable continuous monitoring by the doctor, solution of patients anywhere in the world based on a healthcare monitoring system can be checked. The patients carry a set of body sensors to collect their body parameters.

Rest of the paper is organized as follows. In Section II, Related Work is discussed. Section III proposed system. Section IV, implementation and results. Finally, Section $\mathrm{V}$ concludes the paper along with the further research and references.

\section{Literature Survey}

In ${ }^{1}$ Ravi Kishore Kodali et al. proposed the healthcare monitoring which is implemented to check the temperature of the patient. The Zig Bee mesh protocol is used where the patient 24-hour care records is being monitored. In-hospital records are maintained in the cloud. IoT empowered devices at the same time enrich the quality of care with regular monitoring and collection of data actively and moderate the cost of care and analysis of the same. In $^{2}$ Jasmeet Chhabra et al. proposes the plan and implementation for emergency medical services based on loT health monitoring system. In this project the patient health related problems and healthcare cost is reduced. The collection, recording, analyzing and sharing data streams though the internet which reduce the patient problem of visiting the doctor every time to check the health parameter like heart beat rate, temperature and blood pressure. $\ln ^{3}$ Thirumalasetty Sivakanth et al. presents a reconfigurable sensor network for essential health checking. The possibility of patients collapse and the life-threatening consequences is reduced in content and real-time health monitoring system. The 566 International Conference on Signal, Image Processing Communication and Automationcomplete information of patient is be mechanically obtained by the doctor by NFC technology. Biosensors interfaced with the microcontroller will screen patient's imperative health. If any of the sensor's preset threshold value is overdone above, a sensor's value will be sent to doctors and the patient's caretaker through message. $\operatorname{In}^{4} \mathrm{Y}$. T. Zhan et al. presents the implementation of telehealth systems for elderly population and discussion on various chronic diseases and its importance. They discussed in detail about wearable technology for remote health care system. $\ln ^{5} \mathrm{~A}$. Murray et al. presents the planning of modern medicine, effective and safe use of healthcare technology as essential for any healthcare system. Concerns about medical equipment care have been raised up. There is need to discuss the progress of health care system, In this paper significant progress in the implementation of the healthcare system is proposed. Also the lack of medical equipment safety measures and the protective steps that need to be taken care to improve the quality of healthcare is discussed. $\mathrm{In}^{6}$ Saed Tarapiah et al. presents the paper which guarantees to decrease the cost of the system and overall improvement in the quality of health care services. It is a system that can measure heart rate and body temperature and communicate with them in case of accidental behavior to manage medical personnel using GSM, GPS and web technologies to achieve immediate action to save the patient's life. In ${ }^{7}$ Dr. K.N. Muralidhara and Bhoomika B.K., presents the design for IOT smart healthcare system using the microcontrollers. In this, the pulse oximeter, the temperature sensor and the heart rate are designed for the patient and the microcontroller to send data through the wireless network protocol and the data also shows the patient displayed on the LCD screen who knows his health status. The experts can see the 
information that logs the log to the HTML site of the page using IP address and page recovery methods that are so persisted by the information collection. So the continuous patients check framework is composed.

$\operatorname{In}^{8} \mathrm{D}$. Mahesh Kumar, presents health systems based on wireless sensor networks. The wide range of benefits of wireless technology for the medical staff, patients and the continuous monitoring of the community, early detection of abnormal situations and potential knowledge found in the past data inserted all the information collected. The system helps the health care staff to control the complete state of the patient in a separate, real-time and great way. Through the network can reach every node of the patient at any time as long as the network terminal is available. The patient sends a set of sensors to collect their body parameters. The medical staff evaluates the overall condition of each patient and checks the collected values of the nodes. Luciano Tarricone et al., ${ }^{9}$ paper suggests, loT-aware, architecture for tracking of patients, automatic checking, and biomedical devices within nursing institutes and hospitals. Sampada Sathe and Alok Kulkarni, ${ }^{10}$ paper attempts to evaluate and understand the application of IOT in personalized care for the realization of excellence in health care costs within reasonable limits. Here it describes how loT's functions and how to use it in the use of remote sensing technology and wireless technology to achieve health care requirements.

\section{Proposed System}

A health monitoring system consists of several sensors connected to a patient and they communicate the data through the processing unit. In the project,



Fig. 1: Block Diagram
Raspberry $\mathrm{Pi}$ is used as a data aggregator as well as a processor. The patient and doctor smartphone/ computer are used as a monitoring system.

As in figure 1, the sensors system is used to obtain the information or readings from the patient and the reading which is read are converted into signals. These signals are provided for processing to Raspberry $\mathrm{Pi}$, which is the loT module. The $\mathrm{Pi}$ then displays the information on a Monitor and also stores the information over the cloud. This information can be accessed by the doctor on his phone/computer and get the information. If any emergencies, the patient is sent an alert automatically through the mail for medical medication.

The flow diagram of the project is shown in figure 2 , the sensors value are read and displayed on the monitor and stored in the cloud for future use. If blood pressure sensor output is above 120 an alert mail is sent to the patient automatically to consult the doctor.

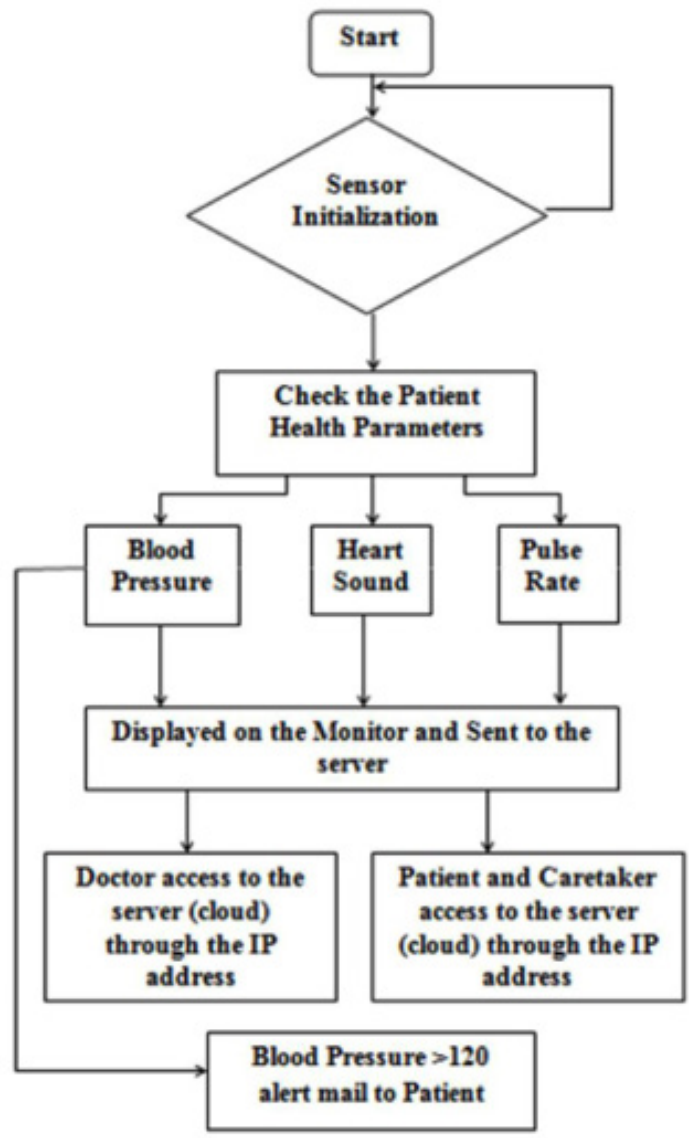

Fig. 2: Flow Diagram 


\section{Implementation and Result}

The kit implementation for Health Monitoring System is shown in the figure 3.The Mouse and Keyboard

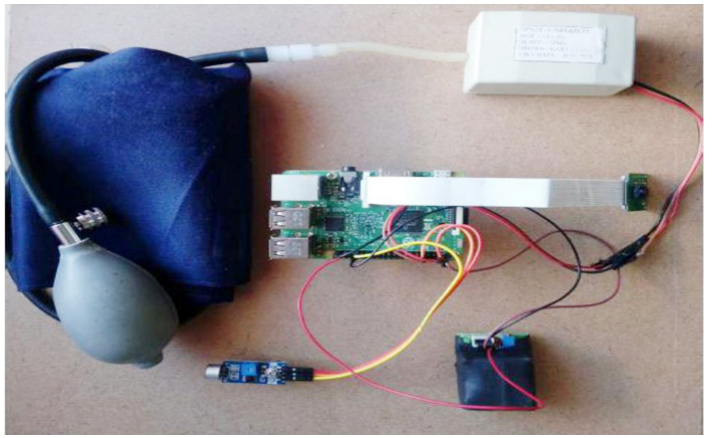

Fig. 3: Kit Implementation for an loT based Health Monitoring



Fig. 4: Display on Monitor after Execution connected to the USB port of $\mathrm{Pi}$ and the Monitor connected to the HDMI video port. The sensors connected to the GPIO pin through which the data from the $\mathrm{Pi}$ is transferred to the server and the patient can monitor the data on the monitor.

Figure 4 shows the display of the health monitoring system on the patient's monitor. After the use of the pulse rate, blood pressure and heart sound sensor, the digital output from the sensor through the $\mathrm{Pi}$ is displayed on the Monitor.

The Pi camera output is displayed on the server, the IP address of the server is same as the IP address of the raspberry pi. The camera output is shown in figure 5 .



Fig. 5: Pi Camera output

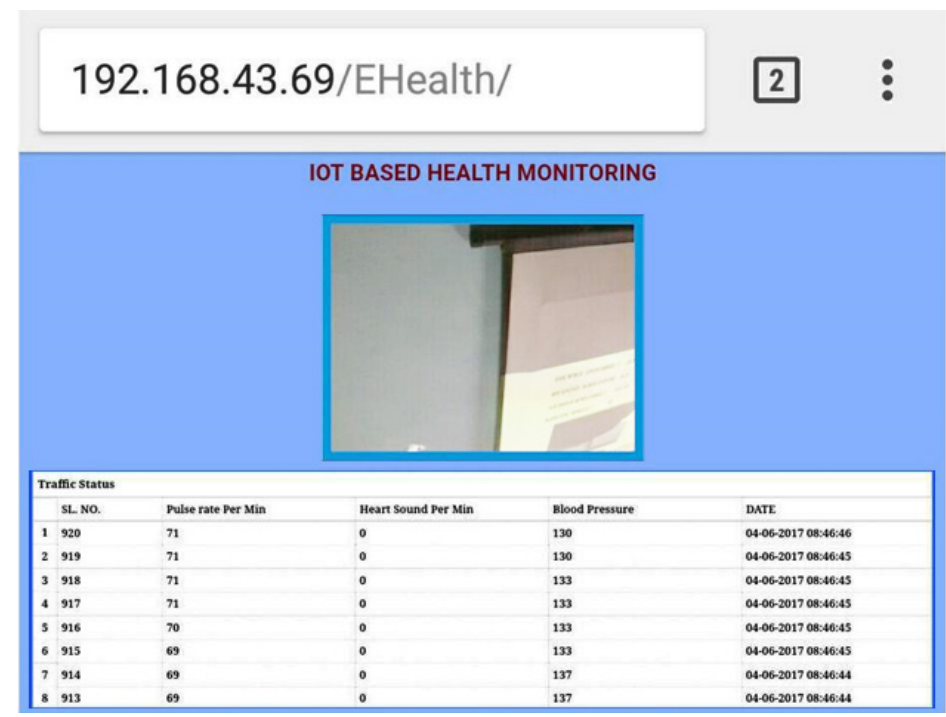

Fig. 6: Server output on Monitor 
The Sensors output is displayed on the server, the IP address of the server is same as the IP address of the raspberry pi which is shown in figure 6 . This is data base where the patient's health report is stored for future requirement by the doctor and the patient. If the patients' blood pressure $>120$ an alert mail is sent to the patient by the doctor for the medial medication.

\section{Conclusion}

IoT Technology is an integration of various technologies which enables different devices and objects to interact with each other and use different network technologies. The proposed system gives better and effective health care services to patients and the information collected is networked worldwide through internet and communication devices in turn connected to cloud services and doctors can use this data and provide a quick and effective solution. The proposed model is a well equipped system where the doctor can check his patient anywhere, anytime. Emergency alert e-mail is sent to the patients if the threshold value is reached that to consult the doctor. This system is helpful for patients who are advised for the complete bed rest and the paralyzed patients, where the doctor can physical monitored the patient from the home with the help of the Pi camera which is used in the system. The aim of the proposed framework is to adopt a new production of medical systems that can provide health care services for high quality and low-cost patients using this combination of large data analysis, cloud computing, and computing technologies. The enhancement for the designed system will connect more sensors and connect all the objects to the Internet for quick and easy access. Further enhancement of existing model can also be deployed as a mobile application in order to become easy to access the model around the world. The mobile application can be enhanced with the ambulance services, doctor's list, nearby hospitals. The patients who are advised for the complete bed rest and the paralyzed patients can also be monitored and given precautions by the doctors by visual and audio by using the pi camera. The system is implemented for one-to-one access, which can be implemented for many by giving unique id for each member/patient in the home or the hospital.

\section{Acknowledgement}

We are very much thankful to the Management of VISTAS Pallavaram for funding this project.

\section{References}

1. Govinda Swamy, Ravi Kishore Kodali and Boppana Lakshmi," An Implementation of loT for Healthcare", IEEE Recent Advances in Intelligent Computational Systems (RAICS) 10-12 December 2015.

2. Punit Gupta, Deepika Agrawal, Jasmeet Chhabra, Pulkit Kumar Dhir, "IoT based Smart HealthCare Kit", Jaypee University of Information Technology, International Conference on Computational Techniques in Information and Communication Technologies (ICCTICT), , 2016.

3. Thirumala settee Sivakanthand $\mathrm{S}$. Kolangiammal, "Design of IoT Based Smart Health Monitoring and Alert System", I J C T A, 9(15), 2016, pp. 7655-7661.

4. B. Tai and Hung K., Y.T. Zhan, "Wearable medical devices for tele-home healthcare," IEMBS'04. 26th Annual International Conference of the IEEE, vol. 2, pp. 53845387, 2004.
5. A. Murray and Khambete N.D., "National efforts to improve healthcare technology management and medical device safety in India," 7th International Conference on, IET, pp. 1-5, 2012.

6. Shadi Atalla, Kahtan Aziz, Salah Haj Ismail and Saed Tarapiah," Smart Real-Time Healthcare Monitoring and Tracking System using GSM/GPS Technologies", 2016 3rd MEC International Conference on Big Data and Smart City.

7. Dr. K.N. Muralidhara and Bhoomika B.K," Secured Smart Healthcare Monitoring System Based on IoT", International Journal on Recent and Innovation Trends in Computing and Communication. Volume: 3 Issue: 7.

8. D. Mahesh Kumar, "Healthcare Monitoring System Using Wireless Sensor Network" Int. J. Advanced Networking and Applications Volume:04 Issue:01 Pages:1497-1500 (2012). 
9. Luciano Tarricone, Luca Mainetti, Luca Catarinucci, Danilo de Donno,Maria Laura Stefanizzi, Luigi Patrono and Luca Palano," An IoT-Aware Architecture for Smart Healthcare Systems", IEEE Internet of Things Journal, Vol. 2, No. 6, December 2015.
10. Sampada Sathe and Alok Kulkarni," Health care applications of the Internet of Things: A Review" International Journal of Computer Science and Information Technologies (IJCSIT), Vol. 5 (5), 2014, 6229-6232. 